www.cya.unam.mx/index.php/cya

Contaduría y Administración, 64 (4), Especial Gobierno Corporativo, 2019, 1-20

\title{
¿Son las prácticas de gobierno corporativo y de earnings management relevantes para el valor de las empresas en México?
}

Are corporate governance and earnings management practices relevant to company value in Mexico?

\section{Isela Alanís Aguilar, Martha del Pilar Rodríguez Garcíåz*, Klender Aimer Cortez Alejandro}

Facultad de Contaduría Pública y Administración, Universidad Autónoma de Nuevo León, México

Recibido el 7 de marzo de 2018; aceptado el 4 de junio de 2019

Disponible en Internet el: 30 de julio de 2019

\begin{abstract}
Resumen
Esta investigación analiza si el gobierno corporativo (conjunto de relaciones entre los diferentes participantes de la empresa) y el earning management (representa la intervención en el proceso de elaboración de la información financiera) tienen impacto en el valor de las empresas en México. La investigación se realizó durante el periodo del 2008 al 2017 utilizando una muestra de 50 empresas listadas en la Bolsa Mexicana de Valores, la metodología empleada fue el método de cuantiles por lo que analizamos el impacto de grandes empresas agrupándolas en pequeñas, medianas y grandes de acuerdo a su tamaño relativo medido por la razón Q de Tobin. Nuestros resultados muestran que existe un impacto negativo del earning management sobre el valor de la empresa en todos los tamaños de empresa medidos por la razón de Q de Tobin. Estos hallazgos, indican una perspectiva oportunista de la gestión de resultados en empresas mexicanas de diferente tamaño. Mientras que el impacto del gobierno corporativo solo se presenta en empresas de tamaño medio. Asimismo, encontramos evidencia de una mayor manipulación en época de crisis, en particular en el 2008, en empresas pequeñas y grandes. Con relación al impacto sectorial se observa que para los percentiles del 25th y 50th el sector industrial, consumo frecuente y telecomunicaciones tienen más impacto sobre la Q de Tobin que el sector de materiales mientras que el sector salud tiene mayor impacto que materiales sobre el valor de la empresa en todos los percentiles.
\end{abstract}

Código JEL: M41, G30, O16

Palabras clave: Gobierno corporativo; Earning management; Bolsa mexicana de valores; Regresión cuantílica

\footnotetext{
* Autor para correspondencia Correo electrónico martha.rodriguezgc@uanl.edu.mx (M. del P. Rodríguez García).

La revisión por pares es responsabilidad de la Universidad Nacional Autónoma de México.

http://dx.doi.org/10.22201/fca.24488410e.2020.1965

0186- 1042/@ 2019 Universidad Nacional Autónoma de México, Facultad de Contaduría y Administración. Este es un artículo Open Access bajo la licencia CC BY-NC-SA (https://creativecommons.org/licenses/by-nc-sa/4.0/)
} 


\begin{abstract}
This research is analyzed if corporate governance (we considering corporate governance as the set of relationships between the different participants of the company) and earning management (intervention in the process of preparing financial information) have an impact on the value of companies in Mexico. The research was conducted during the period from 2008 to 2017, using a sample of 50 companies listed on the Mexican Stock Exchange, the methodology used was the quantile method, so we analyzed the impact of large companies dividing them into small, medium and large firms with Tobin's Q ratio. Our results show that earning management had a negative impact in the value of the company in all company sizes measured by Tobin's $Q$ ratio, which indicates an opportunistic perspective of the management of results in Mexican companies. While the impact of corporate governance only occurs in medium-sized companies. We also find evidence that manipulation is greater in crisis period in small and large companies. Regarding the sectorial impact, it is observed that for the 25th and 50th percentiles the industrial sector, frequent consumption and telecommunications have more impact on Tobin's Q ratio than the materials sector, while the health sector has a greater impact than materials on the value of the company in all percentiles.
\end{abstract}

JEL code: $M 41$, G30, 016

Keywords: Corporate governance; Earning management; Mexican stock exchange; Quantile regression

\title{
Introducción
}

Los escándalos financieros condujeron a un incremento en la transparencia corporativa y una mejora en los sistemas de gobierno corporativo. Los principios de gobierno corporativo de la OCDE (2015) establecen que el gobierno corporativo debe estar alineado a la orientación estratégica de la empresa con el seguimiento efectivo de la gestión y responsabilidad del consejo administrativo. Un sistema efectivo de gobierno corporativo requiere de adecuados niveles de transparencia que reduzcan la asimetría de información entre los gestores y stakeholders de la empresa (Melis, 2004).

En un contexto de interés público surge la incertidumbre de si la aplicación de las medidas de gobierno corporativo promovidas por los códigos de buenas prácticas en entornos empresariales con características diferentes produce resultados similares. Cabe mencionar que mientras en el contexto de Estados Unidos el uso de las medidas de gobierno corporativo promovidas por los códigos es obligatorio para las empresas que cotizan en bolsa, en el contexto europeo se ha optado mayoritariamente por la adopción voluntaria de tales recomendaciones (García y Gill, 2005).

Actualmente el gobierno de Reino Unido ha realizado reformas en relación a los principios de gobierno corporativo (UK Corporate Governance Code, 2017), enfatizando la manera en que las empresas deben ser dirigidas por una junta efectiva que sea colectivamente responsable del éxito a largo plazo de la empresa. En Lombardi (2019) para el caso italiano menciona que se debe implementar un modelo integrado de gobierno corporativo sostenible dirigido a prevenir corrupción. De la misma forma, en Di Pietra y Melis (2016) mencionan que la corrupción de los administradores de las empresas es un problema cada vez más crítico a nivel nacional e internacional y afecta el desarrollo económico y financiero de las empresas. En muchos casos, la corrupción ha sido reconocida como una forma aceptable de comportarse en la conducta empresarial y la gestión en países específicos. La instrumentación del gobierno corporativo debe estar guiada por algo más que requisitos reglamentarios o legales, esto es, un dimensión ética (Cormier y Magnan, 2017).

Ha habido un creciente interés en la comprensión de cómo funcionan las instituciones y las empresas en contextos donde la propiedad familiar está muy extendida en mercados emergentes (Witt y Redding, 2013). De acuerdo a Chong y López de Silanés (2007) en América Latina la protección al inversionista es débil y sus mercados están poco desarrollados. México tiene historia de una mala protección a los inversionistas, siendo su mercado de valores uno de los más pequeños y menos desarrollados del mundo (Chong, et al., 2009).

Para que el gobierno corporativo tenga impacto positivo en el valor de mercado se tienen que presentar dos condiciones (Chong-En et al., 2006). En primer lugar, un buen gobierno debe aumentar los retornos a los accionistas de la empresa y en segundo lugar el mercado de valores debe ser lo suficientemente eficiente que los precios de las acciones 
reflejen los valores fundamentales. Aunque, estos mismos autores mencionan que estas condiciones tienen más probabilidades de ser válidas en los mercados maduros, pero no está claro si también son válidos en los mercados emergentes.

Por otra parte, el earning management ${ }^{2}$ ocurre cuando los gerentes usan el juicio en la información financiera y en la estructuración de transacciones para alterar los informes financieros y uno de los incentivos de las empresas para manipular la información son el efecto en el valor de la empresa (Healy y Wahlen, 1999).

Existe un gran interés por parte de académicos y profesionales de conocer el impacto del gobierno corporativo y del earning management en el valor de la empresa para diferentes áreas de interés y países. Muchos estudios de este tema se encuentran en Europa, Estados Unidos, Latinoamérica y Asia (Drobetz, et al., 2003; Bauer et al., 2004; Beiner et al., 2006; Garay y González, 2008; Chong y López de Silanés, 2007; Huddart y Louis, 2011; Cohen et al., 2011; Gholami et al., 2012; Tangjitprom, 2013). Nosotros deseamos conocer el impacto de este fenómeno en México.

Este estudio contribuye al debate de si en México el gobierno corporativo y el earning management afectan el valor de las empresas durante el periodo 2008-2017. Por ello, el objetivo de esta investigación sería determinar si en México el gobierno corporativo y la manipulación afectan al valor de la empresa en México. Nuestros principales hallazgos muestran que en México en empresas públicas existe manipulación oportunista en diferentes tamaños de empresas medidas por la Q de Tobin y que los efectos son negativos sobre el valor de la empresa. Por otra parte encontramos que el efecto del gobierno corporativo se presenta en empresas de tamaño medio medido a través de la ratio de $\mathrm{Q}$ de Tobin sobre el valor de la empresa. Por último, encontramos evidencia que en periodos de crisis se presenta mayor manipulación en empresas pequeñas y grandes.

El siguiente trabajo de investigación se encuentra dividido en cinco secciones, en primer lugar, se presentan el marco teórico del gobierno corporativo y del earning management, en segundo lugar, una revisión de la literatura en donde mencionamos los principales resultados de investigaciones en Europa, Estados Unidos, Latinoamérica y Asia posteriormente la metodología en donde presentamos los modelos, la hipótesis y la muestra y por último los resultados y conclusiones.

\section{Marco teórico}

\section{Gobierno corporativo y el Earning Management}

La teoría de la agencia surge en Estados Unidos y Reino Unido, siendo el contexto legal e institucional de estos países favorable a la aplicación eficiente de los contratos de agencia (Peng, 2003; Young et al., 2008). El concepto de empresa pública que tiene un enfoque centrado en el accionista y su desempeño es evaluado en el mercado de valores (Davis, 2005). De acuerdo a estudio de Reyna, et al., (2012), la teoría de la agencia es la más importante base teórica que examina y explica la relación entre la estructura de la propiedad y la estructura financiera.

En las economías desarrolladas, debido a que la propiedad y el control a menudo se separan y los mecanismos legales protegen los intereses de los propietarios, los conflictos de gobierno que reciben la mayor parte de la atención son los conflictos de principal-agente. Hay conflicto entre administradores (principal) y accionistas (agente) debido a las diferentes necesidades de cada uno (Jensen y Meckling, 1976). Cuando los gerentes no son los dueños de la empresa, pueden tener incentivos particulares que los alejen de la maximización del valor de la empresa y de los intereses de los accionistas

\footnotetext{
${ }^{2}$ En la literatura también se le identifica con los términos de manipulación contable, gestión de resultados, y manipulación de resultados.
} 
(Berle y Means, 1932; Coase, 1937; Jensen y Meckling, 1976; Fama, 1980; Fama y Jensen, 1983). Es necesario adoptar mecanismos para alinear los intereses entre el principal y el agente (Fama, 1980, Jensen y Meckling, 1976).

En economías emergentes, el contexto institucional y un estado de derecho débil hace que la aplicación de un buen gobierno sea más costosa y problemática (North, 1990; Wright et al., 2005; Dharwadkar et al., 2000). Esto resultado de la concentración de la propiedad de la empresa en uno o pocos dueños (Dharwadkar et al., 2000). En Morck et al. (2005) y Young, et al. (2008) mencionan que la propiedad concentrada y la falta de mecanismos efectivos de buen gobierno, resulta en conflictos más frecuentes entre los accionistas controladores y los accionistas minoritarios. Esto ha llevado al desarrollo de una nueva perspectiva sobre gobierno corporativo, conocida como modelo principal-principal de las empresas. Este enfrentamiento de los accionistas controladores contra los accionistas minoritarios a menudo resulta en la expropiación del valor de los accionistas minoritarios, a los accionistas mayoritarios o de control (Shleifer y Vishny, 1997).

Lefort (2003) hace mención que gobierno corporativo consiste en el conjunto de relaciones que se establecen entre los diferentes participantes en la empresa con el fin de garantizar que cada uno reciba lo que es justo. El gobierno corporativo constituye un elemento clave para aumentar la eficacia económica y potenciar el crecimiento, así como para fomentar la confianza de los inversores (OCDE, 2004).

Ball y Brown (1968) y Beaver (1968), suelen señalarse como los pioneros en analizar la respuesta del mercado de capitales a la información contable. Healy y Wahlen (1999) clasifican los incentivos de las empresas para manipular la contabilidad en tres grupos: incentivos de valoración, incentivos contractuales e incentivos políticos y gubernamentales. García et al. (2005) identifican dos tipos de manipulación la contable y la real. Schipper (1989) define la práctica de earning management como la intervención en el proceso de elaboración de la información financiera y contable, con el propósito de obtener algún beneficio propio.

Resulta fundamental establecer la relación que existe entre las prácticas de buen gobierno corporativo y las prácticas de earning management, dado que los principios del gobierno corporativo también van encaminados a establecer una mayor fiabilidad y transparencia de la información financiera de las empresas. En este contexto, los estudios previos de esta relación se han centrado fundamentalmente en dos aspectos: 1) la composición óptima del consejo de administración y el papel de los consejeros no ejecutivos; y 2) el efecto de la creación y composición de las comisiones delegadas del consejo, especialmente el comité de auditoría. En estas líneas de trabajo desarrolladas en el contexto anglosajón, se ha documentado el papel de las prácticas contables manipuladoras (contabilidad "creativa") que desempeñan tanto los consejeros

independientes, nombrados para representar los intereses de los pequeños accionistas, como de los comités de auditoría independientes (García y Gill, 2005).

En general, las acciones derivadas del gobierno corporativo, deben ir encaminadas a eliminar prácticas de contabilidad manipulada y fraudes en la operación de la empresa. En ocasiones, los intereses de los gerentes los llevan a desarrollar conductas oportunistas en las que la información contable juega un papel importante y en consecuencia puede convertirse en objeto de manipulación, lo que reduce la calidad de la misma. En este sentido, el buen gobierno corporativo debe perseguir la restricción de la práctica de earning management para garantizar la transparencia y calidad de la información. (Callao et al., 2008).

\section{Revisión de la literatura}

Existe estudios aplicados en economías desarrolladas (La Porta et al., 2002) y europeas (Bauer et al. 2004; Renders y Gaeremynck, 2012), que utilizan diferentes muestras de países para encontrar el efecto del gobierno corporativo en el valor de la empresa. En La Porta et al. (2002) durante 1995 y 1996 encontraron una mejor valoración de las empresas en 27 países con mejor protección de accionistas minoritarios. Por otra parte, en Bauer et al. (2004) y Renders y Gaeremynck (2012) 
encuentran que existe una relación positiva entre gobierno corporativo y valor de la empresa, aunque en el primer estudio la relación se debilita con el efecto país.

En Europa oriental Klapper y Love (2004) y Gompers et al. (2003) argumentan que un mejor gobierno corporativo está asociado con un mayor valor de mercado. Asimismo, Gompers et al. (2003) señala que las empresas con fuertes derechos de los accionistas tienen alto valor de mercado. En investigaciones de países desarrollados se encuentran estudios con hallazgos mixtos entre la relación de gobierno corporativo y valor de la empresa. En Alemania, Drobetz, et al. (2003) considerando 30 variables, divididas en cinco categorías ${ }^{3}$ y en Suiza Beiner et al. (2006) considerando 38 atributos de gobierno corporativo demuestran que las empresas con mejor gobierno corporativo tienden a recibir valoraciones más altas. Aunque en Canadá, Klein et al. (2005) utilizando un índice de gobierno corporativo ${ }^{4}$ muestran resultados donde no se encuentra ninguna evidencia sobre la relación entre gobierno corporativo y el valor de la empresa.

En países emergentes, Black (2001) en Rusia; Mousa y Desoky (2012) en Bahrain; y Nazir y Afza (2018) en Paquistán, encuentran que el comportamiento del gobierno corporativo tiene gran efecto sobre el valor de la empresa. En el último estudio, también informan que el comportamiento de los gerentes es oportunista hacia la gestión de las ganancias. Finalmente, Chong-En et al. (2006) en China utilizando una muestra de 1,004 empresas durante 2000 encuentran una relación inversa entre el índice de gobierno corporativo y el valor de la empresa.

Estudios realizados en Latinoamérica también han examinado la relación entre gobierno corporativo y valor de la empresa, aunque existen pocos estudios. En Venezuela, Garay y González (2008) y en México, Chong y López de Silanés (2007) a principios del 2000 examinaron la relación entre gobierno corporativo y valor de la empresa para evaluar las prácticas de gobierno poco estudiadas en estos países. Construyendo un índice de gobierno corporativo para 33 empresas listadas en la Bolsa de Caracas en el año de 2004 y para 159 empresas en el caso de México. Los hallazgos nos muestran una relación positiva y significativa entre el índice de gobierno corporativo y el valor de las empresas.

En resumen podemos decir que estudios en Europa, Latinoamérica y Rusia, existen una gran cantidad de estudios que encuentran asociaciones positivas entre el gobierno corporativo y el valor de la empresa, para estudios de empresas que cotizan en las Bolsa de Valores (Black, 2001; La Porta et al., 2002; Bauer et al., 2004; Drobetz et al., 2003; Gompers et al., 2003; Klapper y Love, 2004; Beiner et al., 2006; López, 2006; Chong y López de Silanés, 2007; Garay y González, 2008; Renders y Gaeremynck (2012). Existen escasas investigaciones que encuentran asociaciones neutras, en Canadá (Klein et al., 2005) y finalmente en China en 1004 empresas encuentran asociaciones negativas entre el gobierno corporativo y el valor de la empresa (Chong-En et al., 2006).

En base a la revisión teórica encontramos un mayor número de estudios con asociaciones positivas en países desarrollados y emergentes. Por lo que nuestra primera hipótesis quedaría como:

$\mathrm{H}_{1}$ : El índice de gobierno corporativo afecta positivamente al valor de la empresa.

\section{Earnings Management y valor de la empresa}

McNichols (2000) identifica tres líneas de investigación utilizadas para detectar earning management los estudios basados en: 1) ajuste por devengo agregado, 2) ajuste por devengo discrecionales específicos, 3 ) distribución del beneficio. Por su parte García et al. (2005) hacen mención a que las dos primeras líneas metodológicas se pueden agrupar conceptualmente en una sola, debido a que permite identificar el instrumento manipulador. Para ello, identifica dos tipos de manipulación

\footnotetext{
3 1) Responsabilidades de gobierno corporativo, 2) Derechos de los accionistas, 3) Transparencia, 4) Manejo y supervisión del consejo de administración, 5) Auditoría.

${ }^{4}$ Este índice fue calculado a través de la suma de cuatro subíndices que miden la: 1) composición del consejo, 2) las políticas de participación accionaria, 3) de compensación, 4) las políticas de derechos de los accionistas y las políticas de divulgación.
} 
I. Alanís Aguilar, et al. / Contaduría y Administración 64(4) Especial Gobierno Corporativo, 2019, 1-20 http://dx.doi.org/10.22201/fca.24488410e.2020.1965

según si ésta afecta únicamente al registro de las transacciones (manipulación contable) o si afecta a las propias transacciones (manipulación real). El modelo acumulaciones agregadas es el más utilizado en la literatura (Jones, 1991).

En trabajos como en Huddart y Louis (2011), Cohen et al. (2011) encontraron una fuerte evidencia de que los administrativos y directores tienden a manipular los componentes de la cuenta de resultados y balance a través de acumulaciones para mantener o maximizar el precio de la acción para el año corriente o para años subsecuentes.

En estudios aplicados en Estados Unidos se encuentran hallazgos mixtos. En primer lugar Magrath y Weld (2002), Jiraporn, et al. (2008) y Gholami et al. (2012) encuentran que los impactos del earning management en el valor de la empresa son positivos. En el primero de ellos mencionan que el earning management puede reducir la volatilidad de las ganancias e incrementar en valor de la empresa. Mientras que en los dos últimos estudios, utilizando muestras grandes, encuentran que el earning management es beneficioso para el valor de la empresa.

En segundo lugar, existen estudios como en Fairfield et al. (2001) y Chan, et al., (2006) en donde la relación entre el earning management y el valor de la empresa es negativa. En el primero de ellos para el periodo de los noventas encuentran que el crecimiento a largo plazo en activos operativos netos, como devengos, tienen una asociación negativa con la rentabilidad de los activos del año entrante. Mientras que en Chan, et al., 2006 mencionan que la imperfección de las normas contables y a errores de medición ocasionan la manipulación oportunista.

En países emergentes los hallazgos son diversos, Lin (2011) realiza un estudio para empresas cotizadas en Taiwán para el periodo de 1997-2007 para una muestra de 277 empresas encontrando una relación no lineal, esto es, los ajustes por devengo discrecionales afectan el valor de la empresa cuando la propiedad del directivo es inferior a 9.67\% (manipulaciones oportunistas), mientas que cuando es superior a 9.67\% se inclinan a gestiones eficientes. En Tailandia, Tangjitprom (2013), para el periodo 2008 a 2011 con una muestra de 1748 observaciones encuentra resultados positivos pero débiles. Por último, en Malasya; Kusuma y Hermuningsih (2013) encuentran relaciones negativas.

Existen estudios en donde la manipulación puede no tener efecto como en Ning (2006) que estudia los beneficios de la práctica de earning management y ha argumentado que ésta no es un fraude, ya que se lleva a cabo dentro de la restricción legal. Las empresas tienen incentivos de reportar ganancias a la baja cuando las ganancias económicas están por encima del nivel deseado. Por otra parte, el earning management puede crear una falsa representación de las ganancias, pero no distorsionar el valor económico de la empresa en términos del activo total, pasivos y capital.

Por último, algunas investigaciones muestran que las empresas llevan a cabo la gestión de resultados como consecuencia de las crisis financieras con el objetivo de solucionar la situación financiera de la empresa (Chia et al. 2007; Dutzi y Rausch, 2016; Lisboa, 2016). En estudios a Eng et al. (2018) encuentran mayor earning management en épocas de crisis como en China y Estados Unidos.

Algunos estudios señalan que el earning management afecta positivamente el valor de la empresa como (Huddart y Louis, 2011; Cohen et al., 2011; Magrath y Weld (2002), Jiraporn, et al. 2008; Gholami et al., 2012 y Tangjitprom, 2013). Otros estudios encuentran relaciones no lineales como Lin (2011) y negativas (Fairfield et al., 2001; Fernandes y Ferreira, 2007; Chan, et al. 2006; Kusuma y Hermuningsih, 2013) y otros neutras (Ning, 2006). Finalmente encontramos que en épocas de crisis se presenta mayor manipulación (Eng et al. 2018).

Derivado de la literatura la práctica de earning management puede presentar diversos comportamientos, debido a que la mayoría de los estudios encuentran asociaciones positivas en países desarrollados y negativas en países emergentes, derivaremos nuestra segunda hipótesis de los trabajos de Lin (2011) y Kusuma y Hermuningsih (2013):

$\mathrm{H}_{2}$ : El earning management afecta negativamente al valor de la empresa.

Asimismo, para determinar que el efecto de la manipulación en crisis basados en el estudio de Eng et al. (2018) nuestra tercera hipótesis quedaría como: 
$\mathrm{H}_{3}$ : El earning management se presenta con mayor intensidad en épocas de crisis que en periodos normales.

Por último, debido a que deseamos determinar si existe un efecto sectorial sobre el valor de la empresa como en estudios desarrollados por Rodríguez et al. (2015), Artikis y Nifora (2011) y Mahmud (2011) en donde encuentran un efecto sectorial en el desempeño financiero. Planteamos una cuarta hipótesis como sigue:

$\mathrm{H}_{4}$ : El sector de la empresa tiene un efecto sobre el valor de la empresa.

\section{Metodología}

La investigación se realizó para el periodo de 2008 a 2017 empleando una muestra de 50 empresas que reportan en la BMV excluyendo a empresas del sector financiero por estar sometidas a una regulación contable distinta al resto de la muestra. Para efectos de este estudio hemos consultado la base de datos de Bloomberg para obtener las series anuales de variables contables y de mercado. En primer lugar para probar $H_{1}, H_{2}$ y $H_{4}$ utilizamos la ecuación (1) con el fin de comprobar si el índice de gobierno corporativo, el earning management y el efecto sectorial tienen impacto en el valor de la empresa en México. Para aplicar este modelo consideramos los ajustes por devengo discrecionales usando la versión modificada del modelo de Jones (1991) que es propuesta por Dechow et al. (1995). A continuación se representa el modelo 1 como:

QTobin $_{i t}=\beta_{0}+\beta_{1} A B S A D_{i t}+\beta_{2} I G C_{i t}+\beta_{3} D E_{A C T_{i t}}+\beta_{4}$ Tamaño $_{i t}+B_{5}$ Industrial $_{i t}+B_{6}$ Cons. Frec $_{i t}+$ $B_{7}$ Bienesnobas $_{i t}+B_{8}$ Tele $_{i t}+B_{9}$ Salud $_{i t}+e_{i t}$

donde:

QTobin $i t=$ valor de mercado de la empresa $i$ del período $t$ dividido por el valor de reposición de los activos de la empresa $i$ en el periodo $t$.

$A B S A D_{i t}=$ valor absoluto de los ajustes por devengo discrecionales $\left(A D_{i t}\right)$ de la empresa $i$ en el momento $t$ deflactado con el activo del periodo $t-1$.

$I G C_{i t}=$ índice de gobierno corporativo de la empresa $i$ en el momento $t$.

$D E \_A C T_{i t}=$ razón de deuda a activos de la empresa $i$ en el periodo $t$.

Tamaño $_{i t}=$ logaritmo natural del activo de la empresa $i$ en el periodo $t$.

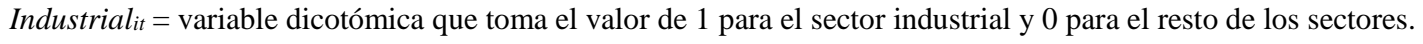

Cons.Frecit = variable dicotómica que toma el valor de 1 para el sector de Bienes de Consumo Frecuente y 0 para el resto de los sectores.

Bienesnobasit $=$ variable dicotómica que toma el valor de 1 para el sector de bienes de consumo no básico y 0 para el resto de los sectores.

Tele $_{i t}=$ variable dicotómica que toma el valor de 1 para el sector de telecomunicaciones y 0 para el resto de los sectores. Salud $_{i t}=$ variable dicotómica que toma el valor de 1 para el sector salud y 0 para el resto de los sectores.

Siguiendo los estudios Chong et al. (2009), Gompers, et al. (2003) se utiliza la medida de Q de Tobin que determina el valor de la empresa dividiendo el valor de mercado de las acciones y el capital contable. Gompers et al. (2003) define la Q de Tobin como el valor de mercado de los activos dividido por el valor de los activos en libros, donde el valor de mercado de los activos más el valor de mercado de las acciones ordinarias menos la suma del valor contable de las acciones ordinarias y los impuestos diferidos.

Para los ajustes por devengo discrecionales se utiliza la versión modificada del modelo de Jones (1991) propuesta por Dechow et al. (1995). Los ajustes por devengo se definen como aquella parte de los ingresos o gastos que no implica 
cobros o pagos. De forma indirecta se calculan por diferencia entre el resultado antes de impuestos y los flujos de caja por operaciones. Hay que distinguir entre los ajustes por devengo no discrecionales $\left(A N D_{i t}\right)$, más difíciles de manipular por la gerencia, y los ajustes por devengo discrecionales $\left(A D_{i t}\right)$, sobre los que la manipulación es más sencilla de hacer.

$$
A T_{i t}=A N D_{i t}+A D_{i t}
$$

Los ajustes por devengo totales se calculan mediante la diferencia entre el resultado de beneficio (pérdidas) de operaciones continuas (RDO) y el cash - flow operativo (CFO) de acuerdo con la siguiente fórmula:

$$
A T_{i t}=R D O_{i t}-C F O_{i t}
$$

Dado que los componentes discrecional y no discrecional de los ajustes por devengo que no son directamente observables y deben ser estimados, usaremos versión modificada del modelo de Jones (1991) propuesta por Dechow, et al. (1995).

$$
\frac{A T_{i t}}{A_{i t-1}}=\alpha_{1} \frac{1}{A_{i t-1}}+\alpha_{2} \frac{\Delta V T A S_{i t}-\Delta \text { Ccobrar }_{i t}}{A_{i t-1}}+\alpha_{3} \frac{{I N M O V_{i t}}}{A_{i t-1}}+e_{i t}
$$

donde:

$A T_{i t}=$ ajustes por devengo totales para la empresa $i$ en el ejercicio $t$

$\triangle V T A S_{i t}=$ variación en ventas de la empresa $i$ en el ejercicio $t$ respecto al $t-1$

$\Delta$ Ccobrar $_{i t}=$ variación en cuentas a cobrar de la empresa $i$ en el ejercicio $t$ respecto a $t-1$

$I N M O V_{i t}=$ cifra de activos fijos brutos para la empresa $i$ en el ejercicio $t$

$e_{i t}=$ término de error para la empresa $i$ en el ejercicio $t$

$A_{i t-1}=$ activo total para la empresa $i$ en el ejercicio $t-1$

La ecuación (4) fue estimada para los periodos 2008-2017, asumiendo que los ajustes por devengo no discrecionales $(A N D)$ se calculan en función de las variaciones anuales en ventas, cuentas por cobrar y activo fijo. El cálculo se realizó en cada una de las observaciones de las empresas de la muestra. Adicionalmente $A_{i t-l}$ se utiliza como deflactor para evitar problemas de heterocedasticidad.

Estimados los ajustes totales $(A T)$ deflactados, se despejó el error, el cual equivale a los ajustes discrecionales $(A D)$ para cada empresa y ejercicio:

$$
A D_{i t}=\frac{A T_{i t}}{A_{i t-1}}-\left(a_{1} \frac{1}{A_{i t-1}}+a_{2} \frac{\Delta V T A S_{i t}-\Delta \text { Ccobrar }_{i t}}{A_{i t-1}}+a_{3} \frac{\text { INMOV }_{i t}}{A_{i t-1}}\right)
$$


$A D_{i \mathrm{t}}=$ ajustes por devengo discrecionales para la empresa $i$ en el ejercicio $t$

$A T_{i t}=$ ajustes por devengo totales para la empresa $i$ en el ejercicio $t$

$\Delta V T A S_{i t}=$ variación en ventas de la empresa $i$ en el ejercicio $t$ respecto al ejercicio $t-1$

$\Delta$ Ccobrar $_{i t}=$ variación en cuentas a cobrar de la empresa $i$ en el ejercicio $t$ respecto al ejercicio $t-1$

$I N M O V_{i t}=$ activo fijo bruto para la empresa $i$ en el ejercicio $t$

$A_{i t-1}=$ activo total para la empresa $i$ en el ejercicio $t-1$

Para incorporar esta variable en la ecuación (1) se utiliza el valor absoluto de los $A D_{i t}$ expresándose como $A B S A D_{i t}$.

Para el índice del gobierno corporativo seguimos una metodología similar a la utilizada por Chong et al. (2009) y Macías y Román (2014), recolectamos información a través del cuestionario de mejores prácticas corporativas para empresas mexicanas que cotizan en la BMV obtenida a través de la información digitalizada de cada empresa para los años 2008-2017. Del cuestionario de mejores prácticas corporativas, se elaboró un índice de gobierno corporativo ${ }^{5}$, sumando un punto por cada respuesta positiva que la empresa cumple con la recomendación del código. Se estandariza el índice entre 0 y 1 dividiendo el número de respuestas positivas por el total del número de respuestas del cuestionario.

Para las variables de control nos basamos en los estudios llevados a cabo por Garay y González (2008) y ChongEn et al. (2006) que incorporan las variables de tamaño y deuda. En Artikis y Nifora (2011) utilizan la variable de control el tipo de sector para determinar el nivel de desempeño financiero. Lo mismo en Gruian (2011) que utiliza el efecto sectorial en trece sectores no financieros. Para la variable sectorial se emplearon seis sectores económicos bajo estudio y consideramos cinco ya que las variables dicotómicas de cada uno de los sectores se explican en función del sector de materiales y eliminamos el problema de colinealidad.

Para probar la $H_{3}$ empleamos la ecuación (1) e incorporamos el efecto de la crisis de 2008 en la ecuación (1) considerando que $A B S A D_{i t}$ puede comportarse diferente en periodos normales y en periodos de crisis, por lo que la ecuación (6), muestra el efecto de la manipulación en periodos normales y de crisis:

$$
\beta_{1}=\beta_{1}^{S C}+\beta_{1}^{C C} \text { Crisis }
$$

donde:

$\beta_{1}=$ manipulación total

$\beta_{1}^{S C}=$ manipulación en periodos normales

$\beta_{1}^{C C}=$ manipulación en períodos de crisis

Crisis = es una variable dicotómica que toma el valor de 0 en periodos normales y de 1 en épocas de crisis

Al sustituir la ecuación (6) en la ecuación (1) el modelo 2 quedaría representado en la ecuación (7) como sigue:

$$
\begin{aligned}
& \text { QTobin }_{i t}=\beta_{0}+\beta_{1}^{S C} A B S A D_{i t}+\beta_{1}^{C C} \text { ABSAD }_{i t} * \text { Crisis }_{i t}+\beta_{2} I_{\text {IGC }}+\beta_{3} \text { DE }_{-} A C T_{i t}+ \\
& \beta_{4} \text { Tamaño }_{i t}+B_{5} \text { Industrial }_{i t}+B_{6} \text { Cons.Frec }_{i t}+B_{7} \text { Bienesnobas }_{i t}+B_{8} \text { Tele }_{i t} \\
& +B_{9} \text { Salud }_{i t}+e_{i t}
\end{aligned}
$$

\footnotetext{
${ }^{5}$ Se consideran cinco categorías generales en el cuestionario de mejores prácticas corporativas: 1) la asamblea de accionistas, 2) el consejo de administración, 3) función de auditoría, 4) función de evaluación y 5) compensación, función de finanzas y planeación.
} 
donde:

$A B S A D_{i t} *$ Crisis $_{i t}=$ representa la interacción de la variable de ajustes por devengo discrecionales $(A B S A D)$ y la una variable dicotómica llamada Crisis que toma valores de 1 en el 2008 y valores de cero en el resto de los periodos.

\section{Método de regresión por cuantiles}

En este estudio deseamos conocer los impactos que el gobierno corporativo y el earning management tienen para diferentes tamaños de empresa en México. Por ello, empleamos la regresión cuantílica. Koenker y Hallock (2001) mencionan que existen ventajas de este método con relación a los tradicionales ya que puede ser más confiable. El modelo se ilustra brevemente de la siguiente manera. Supongamos que hay una especificación lineal para los cuantiles condicionales de $Y_{t}$,

$$
Y_{t}=x_{t} \beta+e_{t}
$$

Donde $Y_{t}$ es la variable dependiente, $x_{t}$ es la variable independiente, $\beta$ es el coeficiente del modelo que se debe estimar, y $e_{t}$ es el término residual. Por lo tanto, el modelo de regresión cuantil estima $\beta$ para diferentes funciones cuantitativas condicionales. Supongamos que la media condicional de $Y$ es $(X)=X^{\prime} \beta$; entonces, la aproximación de los mínimos cuadrados ordinarios calcula la media, $\min _{\mu \in R} \sum_{t=1}^{n}\left(Y_{t}-\mu\right)^{2}$, es decir,

$$
\min _{\beta \in R} \sum_{t=1}^{n}\left(Y_{t}-X^{\prime} \beta\right)^{2}
$$

Resolvemos la ecuación (8) la cual proporciona una estimación de la función mediana (percentil 50). Elegimos $\tau$ para indicar las otras variables cuantitativas. Entonces, la función cuantil se expresa como sigue:

$$
Q_{E}\left(\frac{\tau}{X}\right)=X^{\prime} \beta(\tau)
$$

Por lo tanto, para obtener la estimación de las funciones cuantitativas condicionales, debemos resolver la siguiente ecuación:

$$
\min _{\beta \in R} \sum_{t=1}^{n} \rho_{\tau}\left(Y_{t}-X^{\prime} \beta\right)^{2}
$$

Siguiendo el algoritmo de minimización de la función 10, tenemos:

$$
\min _{\beta}\left[\tau \sum_{E_{t} X^{\prime} \beta}\left|Y_{t}-X^{\prime} \hat{\beta}\right|+(1-\tau) \sum_{Y_{t} \leq X^{\prime} \beta}\left|Y_{t}-X^{\prime} \hat{\beta}\right|\right]
$$


Donde $X^{\prime} \hat{\beta}$ es una aproximación $\tau-t h$ del cuantil condicional de $Y$. Al elegir $\tau$ muy cerca de 0 (1), $X^{\prime} \hat{\beta}$ caracteriza el comportamiento de $Y$ a la izquierda (derecha) de la cola de la distribución condicional. Además, este problema de minimización podría resolverse utilizando el método de programación lineal propuesto por Koenker y D'Orey (1987).

\section{Resultados}

Considerando una muestra de estudio para los años 2008 - 2017 y recopilando la información a través de Bloomberg, la tabla 1 muestra los sectores considerados dentro de la muestra por orden de importancia. En total la muestra contempla 50 empresas, en donde podemos observar que las empresas con mayor participación son el sector industrial y de salud con $26 \%$ de participación respectivamente y con menor salud con $2 \%$.

Tabla 1

Cuadro de empresas por sector

\begin{tabular}{ccc}
\hline Sector & No. de empresas & $\begin{array}{c}\text { Porcentaje de } \\
\text { la muestra }\end{array}$ \\
\hline Industrial & 13 & $26 \%$ \\
Materiales & 13 & $26 \%$ \\
Cons. Frec & 10 & $20 \%$ \\
Bienes no básico. & 7 & $14 \%$ \\
Telecomunicaciones & 6 & $12 \%$ \\
Salud & 1 & $2 \%$ \\
Total & 50 & $100 \%$ \\
\hline
\end{tabular}

Fuente: Elaboración propia

En la tabla 2 se muestra la correlación entre variables independientes de estudio. Realizamos la correlación entre variables independientes para detectar una posible multicolinealidad entre las variables. Los valores son relativamente pequeños lo que indica una baja correlación entre las variables, lo que significa que no hay signos de multicolinelidad.

Tabla 2

Coeficientes de correlación entre variables independientes de estudio

\begin{tabular}{ccccc}
\hline Variable & $A B S A D^{(2)}$ & $I G C^{(3)}$ & $D E_{-} A C T^{(4)}$ & Tamaño $^{(5)}$ \\
\hline$A B S A D$ & 1.00 & -0.13 & 0.05 & -0.12 \\
$I G C$ & 0.13 & 1.00 & 0.012 & -0.02 \\
$D E \_A C T$ & 0.05 & 0.012 & 1.00 & 0.10 \\
Tamaño & -0.12 & -0.025 & 0.10 & 1.00
\end{tabular}

(1) ABSAD: valor absoluto de los ajustes por devengo discrecionales de la empresa $i$ en el momento t deflactado con el activo del periodo $\mathrm{t}-1$.

(2) IGC: índice de gobierno corporativo de la empresa $i$ en el momento t.

(3) $D E \_A C T$ : razón de deuda a activos de la empresa $i$ en el periodo $t$.

(4) Tamaño: logaritmo natural del activo de la empresa $i$ en el periodo $t$.

Fuente: Elaboración propia utilizando E-views

En la tabla 3 determinamos la colinealidad entre las variables empleando el VIF (variance inflation factor) propuesto por Menard (2002), que nos sugiere estimar el modelo de regresión de Mínimos Cuadrados Ordinarios (MCO). EL VIF de la regresión nos muestra cuánto la inflación del error estándar podría ser causada por colinealidad. En nuestro análisis nos arroja valores menores a 10 lo cual nos muestra que no hay problemas de multicolinealidad. 
Tabla 3

VIF de la regresión

\begin{tabular}{lll}
\hline Parámetros & VIF total & $1 /$ VIF \\
\hline$A B S A D^{(1)}$ & 1.07 & 0.9372 \\
$I G C^{(2)}$ & 1.17 & 0.8559 \\
DE_ACT (3) & 1.23 & 0.8156 \\
Tamaño $^{(4)}$ & 1.2 & 0.8334 \\
Industrial $^{(5)}$ & 1.6 & 0.6268 \\
Cons.Frec $^{(6)}$ & 1.59 & 0.6309 \\
Bienesnobas $^{(7)}$ & 1.41 & 0.7111 \\
Tele $^{(8)}$ & 1.45 & 0.6887 \\
Salud $^{(9)}$ & 1.12 & 0.8964 \\
\hline
\end{tabular}

Fuente: Elaboración propia utilizando Stata v12.

Por otra parte, en la tabla 4 se muestran las estadísticas descriptivas de las variables del modelo expuesto anteriormente. La variable que representa la mayor media es tamaño (4.39) y la que representa la menor media es el ABSAD (.05). Por otra parte, la variable que representa mayor desviación estándar es la Q de Tobin (.70), mientras que el ABSAD (.05) representa la menor desviación estándar. El valor de asimetría deberá indicarnos si los valores de la distribución se disponen simétricamente alrededor de la media o bien si tienen mayor media hacia la derecha o hacia la izquierda. Para la variable ABSAD la asimetría es positiva ya que los datos se encuentran por encima del valor de la media aritmética. Mientras que el IGC presenta una asimetría negativa ya que los datos se localizan en los valores menores a la media. Debido a la presencia de curtosis y asimetría en los datos, esto puede indicar datos atípicos y asimetría en la distribución de las variables del estudio. Por lo cual el uso de la metodología de cuantiles es viable en esta investigación.

Tabla 4

Estadísticas descriptivas

\begin{tabular}{rccccc}
\hline Statistic & QTobin $^{(1)}$ & ABSAD & IGC & & DE_ACT \\
Media & 1.51 & 0.05 & 0.91 & 0.29 & Tamaño $^{(5)}$ \\
Des.Est. & 0.70 & 0.05 & 0.06 & 0.17 & 0.60 \\
Asimetría & 1.65 & 3.20 & -2.30 & 0.72 & 0.49 \\
Curtosis & 7.01 & 20.44 & 12.44 & 3.28 & 2.90
\end{tabular}

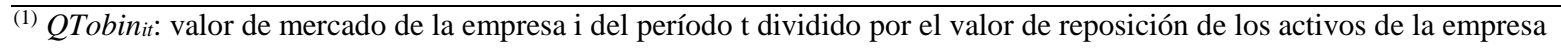
$i$ en el periodo $t$.

(2) $A B S A D_{i t}$ : valor absoluto de los ajustes por devengo discrecionales de la empresa $i$ en el momento $t$ deflactado con el activo del periodo $t$ - 1 .

(3) $I G C_{i t}$ : índice de gobierno corporativo de la empresa $i$ en el momento $t$.

(4) $D E_{-} A C T_{i t}$ : Razón de deuda a activos de la empresa $i$ en el periodo $t$

(5) Tamaño $i$ : Logaritmo natural del activo de la empresa $i$ en el periodo $t$

Fuente: Elaboración propia utilizando E-views

Aplicando el modelo 1, los resultados se muestran en la Tabla 5, examinando la relación entre la práctica de gestión de resultados y el valor de la empresa y determinar si la gestión es oportunista o beneficiosa. En primer lugar, determinaremos el signo de esta relación, si los gerentes administran los resultados para su beneficio y no con el propósito de generar riqueza al accionista, se encontraría una relación inversa entre el grado de gestión de resultados y el valor de la 
empresa. Al hacer el análisis por percentiles, los resultados muestran que existe una relación inversa y significativa entre el earnig management y el valor de la empresa en todos los percentiles (25th, 75thy 50th). Además, este oportunismo o manipulación se mantiene se presenta en un rango de -1.3 a -1.4, lo que indica que el tamaño la manipulación no cambia al cambiar el percentil bajo análisis. La influencia negativa en el resultado sugiere una perspectiva oportunista, consistente con los resultados obtenidos en países emergentes (Lin, 2011 y Kusuma y Hermuningsih, 2013).

Tabla 5

Datos Panel y Regresión cuantil por sectores

\begin{tabular}{|c|c|c|c|c|c|c|}
\hline \multirow{2}{*}{$\frac{\text { Variable }}{A B S A D^{(1)}}$} & \multirow{2}{*}{$\frac{25^{\text {th }}}{-1.448}$} & \multirow{2}{*}{$\frac{\text { percentile }}{(-2.9479) *}$} & \multicolumn{2}{|c|}{ 50th percentile } & \multicolumn{2}{|c|}{$75^{\text {th }}$ percentile } \\
\hline & & & -1.3837 & $(-3.1894)^{*}$ & -1.3222 & $(-1.7169) * *$ \\
\hline$I G C^{(2)}$ & 0.0099 & $(0.0336)$ & 0.5858 & $(1.8721)^{* *}$ & 1.0386 & $(1.3181)$ \\
\hline$D E \_A C T(3)$ & -0.1811 & $(-1.2136)$ & -0.2951 & $(-1.7752) * *$ & -0.8359 & $(-2.6416) *$ \\
\hline Tamaño $o^{(4)}$ & 0.1236 & $(3.0620)^{*}$ & 0.126 & $(3.1736)^{*}$ & 0.2761 & $(2.8380) *$ \\
\hline Industrial $^{(5)}$ & 0.1973 & $(2.8848) *$ & 0.1742 & $(2.5840) * *$ & 0.5905 & $(2.7560) *$ \\
\hline Cons.Frec ${ }^{(6)}$ & 0.3989 & $(6.7991) *$ & 0.3236 & $(4.5941)^{*}$ & 0.2019 & $(1.6644)^{* *}$ \\
\hline Bienesnobas $^{(7)}$ & 0.0826 & $(1.0835)$ & 0.1301 & $(1.2680)$ & 0.2255 & $(1.3641)$ \\
\hline Tele $^{(8)}$ & 0.3589 & $(4.3626)^{*}$ & 0.3374 & $(3.3670) *$ & 0.4448 & $(-1.3082)$ \\
\hline Salud ${ }^{(9)}$ & 0.9415 & $(5.2711) *$ & 0.8617 & $(8.5156)^{*}$ & 0.5159 & $(3.1587) *$ \\
\hline $\operatorname{Cons}^{(10)}$ & 0.4821 & (1.4947) & 0.2363 & $(0.7333)$ & -0.365 & $(-0.4026)$ \\
\hline$R$-squared & & 0.103887 & & 0.11088 & & 0.069856 \\
\hline
\end{tabular}

Nota: *p-value $<0.10, * *$ p-value $<0.05 t$ values en paréntesis

(1) $A B S A D_{i t}$ : valor absoluto de los ajustes por devengo discrecionales de la empresa $i$ en el momento $t$ deflactado con el activo del periodo $t-1$.

(2) $I G C_{i t}$ : índice de gobierno corporativo de la empresa $i$ en el momento $t$.

(3) $D E \_A C T_{i t}$ : Razón de deuda a activos de la empresa $i$ en el periodo $t$

(4) Tamaño $i t$ : Logaritmo natural del activo de la empresa $i$ en el periodo $t$

${ }^{(5}$ Industrialit: variable dicotómica que toma el valor de 1 para el sector industrial y 0 para el resto de los sectores.

(6) Cons.Frec it: variable dicotómica que toma el valor de 1 para el sector de Bienes de Consumo Frecuente y 0 para el resto de los sectores.

(7) Bienesnobasit: variable dicotómica que toma el valor de 1 para el sector de bienes de consumo no básico y 0 para el resto de los sectores.

${ }^{(8)}$ Tele: variable dicotómica que toma el valor de 1 para el sector de telecomunicaciones y 0 para el resto de los sectores.

${ }^{(9)}$ Salud: variable dicotómica que toma el valor de 1 para el sector salud y 0 para el resto de los sectores.

${ }^{(10)}$ Cons: constante de la regresión

Fuente: Elaboración propia utilizando E-views

Mientras que en el caso del gobierno corporativo observamos que las empresas pequeñas o grandes, es decir en los extremos no tiene efecto el gobierno sobre la Q de Tobin. Por otro lado, efecto es positivo y significativo y se presenta en empresas en el percentil 50th, esto es, el efecto del gobierno corporativo solo se presenta en empresas con Q de Tobin alrededor de la mediana. Estos hallazgos son consistentes con el estudio de Garay y González (2008). También nuestros resultados son consistentes con los estudios de Polleti (2011), Chong, et al. (2009) y Klapper y Love (2004) que presentan resultados positivos y significativos, sosteniendo su hipótesis de que las empresas con mejor gobierno corporativo tienen valuaciones más altas.

El signo esperado de la deuda es negativo, pero no significativo en las empresas pequeñas. Estos resultados no son consistentes con Chong, et al. (2009) y Chong-En et al. (2006) en donde encuentran una relación inversa entre deuda y valor de la empresa. Por Por otra parte, los resultados son los esperado en empresas medianas y grandes donde existe una 
relación inversa entre endeudamiento y valor de la empresa. Estos hallazgos son consistentes con las conclusiones del estudio de Frijns et al. (2016).

Para la variable Tamaño, los coeficientes son positivos y significativos a un 5\% en los 3 percentiles y que el efecto de la variable tamaño es mayor entre mayor es la empresa medida con la Q de Tobin. Estos resultados son consistentes con los resultados de Poletti (2011) en donde obtiene que existe una relación directa entre el tamaño y el valor de la empresa. También resulta consistente con los resultados de Jiraporn, et al. (2008), Renders y Gaeremynck (2012). Igual que Kusuma y Hermuningsih (2013).

En relación al efecto sectorial las empresas del sector Salud tiene más impacto en la Q de Tobin que las empresas del sector Materiales en todos los cuantiles, lo mismo sucede con el sector de Comunicaciones y Transportes. Los resultados son consistentes con Artikis y Nifora (2011). Por otra parte el sector Industrial tiene más efecto en las empresas grandes que el sector de Materiales. Estos últimos hallazgos son consistentes con Mahmud (2011) que encuentra que el sector de la ropa de vestir es uno de los más rentables que estaría dentro del industrial.

Por otra parte, estimamos el modelo 2 considerado para determinar el efecto de la crisis de 2008. Los resultados se presentan en la tabla 6. La manipulación se evidencia principalmente en periodo de crisis ya que al interactuar la variable dicotómica de Crisis con la ABSAD el coeficiente es significativo y negativo en empresas pequeñas y grandes a diferencia del coeficiente de ABSAD sin interacción de crisis. Los resultados son similares a los encontrados en trabajos de Lisboa (2016) y Eng et al. (2018). Asimismo, la variable de IGC de gobierno corporativo es positiva en empresas grandes incluyendo el periodo de crisis y en este mismo tamaño de empresas el resto de los coeficientes muestran los signos esperados. En las empresas pequeñas y medianas el gobierno corporativo y la gestión de resultados no muestran efecto sobre la Q de Tobin.

Tabla 6

Datos Panel y Regresión cuantil por sectores y el efecto de la crisis

\begin{tabular}{|c|c|c|c|c|c|c|}
\hline \multirow{2}{*}{$\frac{\text { Variable }}{A B S A D^{(I)}}$} & \multirow{2}{*}{$\frac{25^{\text {th }}}{-0.5809}$} & \multirow{2}{*}{$\frac{\text { percentile }}{(-1.1163)}$} & \multicolumn{2}{|c|}{ 50th percentile } & \multicolumn{2}{|c|}{$75^{\text {th }}$ percentile } \\
\hline & & & -0.9259 & $(-1.6460)$ & -0.9294 & $(-1.2604)$ \\
\hline$A B S A D^{*}$ Crisis $^{(2)}$ & -1.1971 & $(-1.7928) * *$ & -1.0295 & $(1.3561)$ & -1.8003 & $(-2.3150) *$ \\
\hline$I G C^{(3)}$ & -0.071 & $(-0.2312)$ & 0.5511 & $(1.5636)$ & 1.176 & $(2.7171)^{*}$ \\
\hline$D E \_A C T^{(4)}$ & -0.1689 & $(-1.2089)$ & -0.3582 & $(-2.1622) *$ & -0.888 & $(-3.1672) *$ \\
\hline Tamaño(5) & 0.121 & $(3.2598) *$ & 0.1183 & $(2.9877)^{*}$ & 0.2643 & $(2.8525) *$ \\
\hline Industrial ${ }^{(6)}$ & 0.161 & $(2.5346)^{*}$ & 0.1521 & $(2.2797)^{*}$ & 0.5613 & $(2.9832) *$ \\
\hline ConsFrec $^{(7)}$ & 0.3744 & $(6.3226)^{*}$ & 0.3184 & $(4.6196)^{*}$ & 0.2203 & $(1.8736)^{* *}$ \\
\hline Bienesnobas $^{(8)}$ & 0.043 & $(0.5964)$ & 0.1304 & $(1.2678)$ & 0.2766 & $(1.8943)^{* *}$ \\
\hline Tele $e^{(9)}$ & 0.2847 & $(3.2242)^{*}$ & 0.3479 & $(3.4671)^{*}$ & 0.4069 & $(2.3335) *$ \\
\hline Salud $^{(10)}$ & 0.9391 & $(5.3511)^{*}$ & 0.8693 & $(8.1254)^{*}$ & 0.491 & $(3.1692)^{*}$ \\
\hline Cons $^{(11)}$ & 0.567 & $a(1.8162)$ & 0.3078 & $(0.8738)$ & -0.4278 & $(-0.7869)$ \\
\hline$R$-squared & & 0.108789 & & 0.11363 & & 0.074703 \\
\hline
\end{tabular}


Nota: $*$ p-value $<0.10, * *$ p-value $<0.05 \mathrm{t}$ values en paréntesis

(1) $A B S A D_{i t}$ : valor absoluto de los ajustes por devengo discrecionales de la empresa $i$ en el momento $t$ deflactado con el activo del periodo $t-1$.

(2) $A B S A D^{*}$ Crisis: representa la interacción de la variable de ajustes por devengo discrecionales $(A B S A D)$ y la una variable dicotómica llamada Crisis que toma valores de 1 en el 2008 y valores de cero en el resto de los periodos $t$.

(3) $I G C_{i t}$ : índice de gobierno corporativo de la empresa $i$ en el momento $t$.

(4) $D E \_A C T_{i t}$ : Razón de deuda a activos de la empresa $i$ en el periodo $t$

(5) Tamañoit: Logaritmo natural del activo de la empresa $i$ en el periodo $t$

(6) Industrialit: variable dicotómica que toma el valor de 1 para el sector industrial y 0 para el resto de los sectores.

(7) Cons.Frec it: variable dicotómica que toma el valor de 1 para el sector de Bienes de Consumo Frecuente y 0 para el resto de los sectores.

(8) Bienesnobasit: variable dicotómica que toma el valor de 1 para el sector de bienes de consumo no básico y 0 para el resto de los sectores.

${ }^{(9)}$ Tele: variable dicotómica que toma el valor de 1 para el sector de telecomunicaciones y 0 para el resto de los sectores.

${ }^{(10)}$ Salud: variable dicotómica que toma el valor de 1 para el sector salud y 0 para el resto de los sectores.

${ }^{(11)}$ Cons: constante de la regresión

Fuente: Elaboración propia utilizando E-views

Para probar la robustez en el modelo 1 sustituimos la variable dependiente, la Q de Tobin por el rendimiento sobre activos (ROA), métrica de desempeño validada en otros estudios (Chong y López de Silanés, 2007). Estimamos los modelos para los percentiles 25, 50 y 75 y encontramos que los resultados y la prueba de bondad de ajuste (R2) son similares a los obtenidos con la variable original dependiente ( $\mathrm{Q}$ de Tobin). Por otra parte para probar la robustez en las variables independientes (ABSAD y el IGC), incorporamos el indicador de gobierno corporativo que provee Bloomberg. Los resultados muestran que el modelo no cambia significativamente, aunque la R2 es más alta con el índice de gobierno corporativo que se consideró inicialmente. Con estas pruebas de robustez, mostramos que el modelo no cambia significativamente al cambiar la variable dependiente o al cambiar la variable independiente con variables similares a las consideradas en los modelos originales.

\section{Conclusiones}

La normativa contable no es solamente el resultado de un proceso económico, sino que también de un proceso político, y como la contabilidad con base de devengado involucra criterios subjetivos acerca de los flujos de caja futuros, deja espacio para que los productores de la información contable (ejecutivos, empresas) y los cuerpos normativos y controladores (Cortes, reguladores, directorios, gremios, etc.) apliquen criterios locales (Ball y Shivakumar, 2005). Es por ello, que en esta investigación deseábamos conocer los impactos de la gestión de resultados y de gobierno corporativo para empresas de diferente valor de mercado medido a través de la Q de Tobin.

Para México, esta investigación es importante debido a que pudimos constatar que existe una manipulación oportunista en la BMV para todos los tamaños de percentiles medidos por la Q de Tobin. Para Chong y López de Silanés (2007) nuestra economía mexicana tiene un sistema legal débil. Por lo que fomenta de esta forma la gestión de resultados oportunistas. En este sentido, los inversionistas deberían seguir la información contable y usarla con cierta prudencia para la toma de decisiones. Es recomendable que los tomadores de decisiones se percaten del análisis de la cuenta de resultados y del balance y determinar el buen manejo administrativo de las empresas donde desean invertir. De esta forma pudiéramos aumentar la confianza de los inversionistas en las empresas mexicana y puede ser un factor clave del crecimiento de la inversión y capitalización en México.

Además, este estudio ofrece contribuciones importantes para México en materia de gobierno corporativo ya que encontramos que este impacta al valor de la empresa en empresas de tamaño medio de Q de Tobin. Los hallazgos muestran que las prácticas de gobierno corporativo afectan fuertemente y positivamente el valor de las empresas en México como en estudios similares a nivel internacional (Klapper y Love, 2004) que encuentra esta relación en ambientes débiles. Por otra 
parte, a nivel nacional estudios realizados por Chong y López de Silanés (2007) que comprueban esta misma asociación positiva para el caso mexicano. Lo importante de este hallazgo es que encontramos que el efecto del gobierno corporativo se presenta en empresas medianas y no en los casos extremos, estos resultados son diferentes a los obtenidos por Drobetz, et al. (2003) en donde encuentran que las empresas con alto gobierno corporativo tienden a ser las grandes.

Es importante que las empresas pequeñas y grandes apliquen los controles de gobierno corporativo a fin de contribuir a la transparencia de información de la empresa y con ello impactar positivamente al valor de la empresa. Las limitaciones se concentran en la construcción del índice de gobierno corporativo y en que no presentamos hallazgos en relación a los factores del gobierno corporativo como en Mousa y Desoky (2012) que estudian las características del consejo de administración y estructura de la propiedad encontrando efecto positivo en el valor de la empresa. Lo anterior, nos permite seguir investigando el tema de manera más específica en investigaciones posteriores.

Además, encontramos que en periodos de crisis la manipulación se presenta en mayor medida en las empresas agrupadas en pequeñas y grandes siendo el efecto mayor en las grandes que en las pequeñas. Esta gestión de resultados afecta de manera negativa a las empresas. Asimismo podemos mencionar que según Drobetz, et al. (2003) encuentran que las empresas con alto gobierno corporativo tienden a ser las grandes, en nuestro modelo con el efecto crisis encontramos los mismos hallazgos, aunque también encontramos que son las que más manipulan.

Por último, nuestros hallazgos prueban la necesidad de dedicar esfuerzos adicionales por parte de los administradores a fin de que contribuyan en los mercados de capitales para mejorar la transparencia de sus informes y con ello influir en el valor de la empresa. Además, lo anterior nos permitirá que en México el inversionista confíe en las instituciones, en la información financiera y en buenos controles de gobierno corporativo ayudando de esta forma a que las empresas cuenten con desempeños financieros sostenibles en el tiempo.

\section{Referencias}

Artikis, P. y Nifora, G, (2011). The Industry Effect On The Relationship Between Leverage and Returns. Eurasian Business Review, 1(2), 125-145

Ball, R. y Brown, P. (1968). An empirical evaluation of accounting income numbers. Journal of Accounting Research, 129178, https://doi.org/10.2307/2490232

Ball, R., y Shivakumar, L. (2005). Earnings quality in UK private firms: Comparative loss recognition timeliness. Journal of Accounting and Economics, 39(1), 83-128. http://doi.org/10.1016/j.jacceco.2004.04.001

Bauer, R. Guenster, N., y Otten, R. (2004). Empirical evidence on corporate governance in Europe: The effect on stock returns, firm value, and performance. Journal of Asset Management, 5(2), 91-104. https://doi.org/10.1057/palgrave.jam.2240131

Beaver, W. (1968). The information content of accounting earnings announcements. Journal of Accounting Research, 6792, https://doi.org/10.2307/2490070

Beiner, S., Drobetz, W., Schmid, M. M., y Zimmermann, H. (2006). An integrated framework of corporate governance and firm valuation. European Financial Management, 34, 249-283. https://doi.org/10.1111/j.1354-7798.2006.00318.x

Berle, A. y Means, G. (1932). The modern corporation and private property. Nueva York: MacMillan. https://doi.org/10.2307/3475545

Black, B. (2001). Does Corporate Governance Matter? A Crude Test Using Russian Data, University of Pennsylvania Law Review, 149, 2131-2150. https://doi.org/10.2139/ssrn.252706

Callao, S., Gasca, M. M. y Jarné, J.I. (2008). Gobierno Corporativo y deficiencias de la información Contable. RC-SAR, $10(1), 133-156$ 
Chan, K., Chan, L. Jegadeesh, N. y Lakonishok, J. (2006). Earnings quality and stock returns, Journal of Business, 79, 10411082. https://doi.org/10.1086/500669

Chia, Y., Lapsley, I., y Lee, H. (2007). Choice of auditors and earnings management during the Asian financial crisis. ManagerialAuditing Journal, 22(2), 177-196.

Chong, A. y López de Silanés, F. (2007). Investor protection and corporate governance. Firm level evidence across Latin America. Washington D.C.: Stanford University Press-Inter American Development Bank. https://doi.org/10.1596/978-0-8213-6913-5

Chong, A., Guillén J. y López-de-Silanes, S. (2009). Corporate governance reform and firm value in Mexico: an empirical. Journal of Political Economy Reform, 12(3), 163-188. doi.org/10.1080/17487870903105346

Chong-En, B., Qiao, L., Joe, L., Frank, S., y Junxi, Z. (2006). An Empirical Study on Corporate Governance and Market Valuation in China. Front. Econ.China, 1, 83-111. https://doi.org/10.1007/s11459-005-0011-0

Coase, R. (1937). The nature of the firm. Económica, 4(16), 386-405. DOI: 10.1111/j.1468-0335.1937.tb00002.x

Cohen, L., Marcus A.J., Rezaee, Z. y Tehranian H. (2011). Earnings Guidance, Earning management, and Share Prices 142 Disponible en https://www2.bc.edu/ cohenlj/Guidance\%2008_25_2011.pdf (acceso el 24-May-16).

Cormier, D., Magnan, M. (2017). Does a firm's exposure to ethical failures matter to financial markets? A governance perspective. Journal of Management \& Government, 21 (2), 267-289. https://doi.org/10.1007/s10997-016-93419

Davis, G. F. (2005). New directions in corporate governance. Annual Review of Sociology, 31(1), $143-162$. https://doi.org/10.1146/annurev.soc.31.041304.122249.

Dechow, P.M., Sloan, R.G. y Sweeney, A.P. (1995). Detecting earnings management. The Accounting Review, 70(2), 193225.

Di Pietra, R. y Melis, A. (2016). Governance and corruption: is history repeating itself? Fostering a debate and inviting contributions from a multidisciplinary perspective. Journal of Management \& Government, 20 (4) 689701.https:doi.org/10.1007/s10997-015-9326-0.

Dharwadkar, R., George, G. and Brandes, P. (2000). Privatization in emerging economies: an agency theory perspective. Academy of Management Review, 25, 650-69.

Drobetz, W., Schillhofer, A. y Zimmermann, H. (2003). Corporate governance and expected stock returns: Evidence from Germany. European Financial Management. 10(2), 267-293. DOI: 10.1111/j.1354-7798.2004.00250.x

Dutzi, A., y Rausch, B. (2016). Earnings management beforebankruptcy: A review of the literature. Journal of Accounting and Auditing: Research \& Practice, 2016, 1-21. http://dx.doi.org/10.5171/2016.245891. ID 245891

Eng L., Fang H., Tian X., Yu T., Zhang, H. (2018) Financial crisis and real earnings management in family firms: A comparison between China and the United States, Journal of International Financial Markets, Institutions and Money (In Press). https://doi.org/10.1016/j.intfin.2018.12.008.

Fairfield, P., Whisenant, S. y Yohn T.L. (2001). Accrued Earnings and Growth: Implications for Earnings Persistence and Market Mispricing, SSRN Electronic Journal, DOI:10.2139/ssrn.249311 Available from: Scott Whisenant. Recuperado el: 04 April 2016

Fama, E. (1980). Agency problems and the theory of the firm. Journal of Political Economy, 88 (2), $288-307$. https://doi.org/10.1086/260866 https://doi.org/10.1086/260866

Fama, E. y Jensen, M. (1983). Agency problems and residual claims. Journal of Law and Economics, 26, $327-349$. http://dx.doi.org/10.2139/ssrn.94032 
Fernandes, N. y Ferreira M. (2007). The evolution of Earnings Management and Firm Valuation: A Cross-Country Analysis. EFA 2007 Ljubljana meetings paper, 1-38. http://papers.ssrn.com/sol3/papers.cfm?abstract_id=965636 https://doi.org/10.2139/ssrn.965636

Frijns B., Dodd, O., Cimerova, H. (2016). The impact of cultural diversity in corporate boards on firm performance, Journal of Corporate Finance, 41, 521-541 https://doi.org/10.1016/j.jcorpfin.2016.07.014

Garay, U. y González, M. (2008). Corporate Governance and Firm Value: the case of Venezuela. Journal Copilation, 16(3), 194-209. doi:10.1111/j.1467-8683.2008.00680.x

García B., Gill, B. y Gisbert, A. (2005). La Investigación sobre Earnings managements. Revista Española de Financiación y Contabilidad, XXXIV(127), 1001-1033. https://doi.org/10.1080/02102412.2005.10779570

García, B. y Gill, B. (2005). El Gobierno Corporativo y las Prácticas de Earnings Management: Evidencia Empírica en España, Instituto Valenciano de Investigaciones Económicas, S.A., España, 2-10.

Gholami, S., Nickjoo, M. y Nemati, S. (2012). The study of the relation between Earning management and Long Run Stock Performance, Journal of Basic and Applied Scientific Research, 2(8), 8008 - 8010.

Gompers, P., Ishii J. y Metrick A. (2003). Corporate Governance and Equity. The Quarterly Journal of Economics, 118(1), 107-156. doi: 10.1162/00335530360535162

Gruian, C. (2011). The Financial Performance of European Companies: A Comparative Approach. Annals of the University of Oradea, Economic Science Series 20 (1): 193-200

Healy, P. y Wahlen, M. (1999). A review of the earnings management literature and its implications for standard setting, Accounting Horizons, 13 (4), pp. 365-383. https://doi.org/10.2308/acch.1999.13.4.365

Huddart, S. y Louis, H. (2011). Insider Selling, Earnings management, and the 1990s Stock Market Bubble, SSRN Electronic Journal, 1-58. Disponible en https://www.researchgate.net/publication/228230301 Insider Selling Earning management and the 1990's Stock Market Bubble (acces 24-May-16). https://doi.org/10.2139/ssrn.912214

Jensen, M. y Meckling, W. (1976). Theory of the firm: Managerial behavior, agency costs and ownership structure. Journal of Financial Economics, 3(4), 305-360. https://doi.org/10.1016/0304-405x(76)90026-x

Jiraporn, G., Miller, S. Yoon, y Kim. Y. (2008). Is earnings management opportunistic or beneficial? An agency theory perspective, International Review of Financial Analysis,17, 622-634. https://doi.org/10.1016/j.irfa.2006.10.005

Jones, J. (1991). Earnings management during import relief investigations. Journal of Accounting Research. 29 (2), 193 128. https://doi.org/10.2307/2491047

Klapper, L., y Love, I. (2004), Corporate Governance, Investor Protection, and Performance in Emerging Markets, Journal of Corporate Finance, 10, 703-728. https://doi.org/10.2139/ssrn.303979

Klein, P., Shapiro, D., y Ypung J. (2005). Corporate Governance, Family Ownership and Firm Value: the Canadian evidence. An International review, 38(6), 769-784. https://doi.org/10.1111/j.1467-8683.2005.00469.x

Koenker, R. y Hallock, K., (2001). Quantile regression: an introduction. Journal of Econmics Perspectives, 15(4), $143-156$. https://doi.org/10.1257/jep.15.4.143

Koenker, R.W. y D'Orey, V., (1987). Algorithm as 229: computing regression quantiles. Appl. Stat. 36:383-393. http://dx.doi.org/10.2307/2347802.

Kusuma, D. y Hermuningsih S. (2013). Does Earning management Reduce Firm Value in Malaysia. International Conference on Business and Management 2-4 Octubre 2013, Chiang Mai - Bangkok.

La Porta L., López-de-Silanes, F, y Shleifer, A. (2002). Investor Protection and Corporate Valuation. Journal of Finance, 57(3), 1147-1170. https://doi.org/10.1111/1540-6261.00457 
I. Alanís Aguilar, et al. / Contaduría y Administración 64(4) Especial Gobierno Corporativo, 2019, 1-20 http://dx.doi.org/10.22201/fca.24488410e.2020.1965

Lefort, F. (2003). Gobierno Corporativo: ¿Qué es? Y ¿Cómo Andamos por Casa?, Cuadernos de Economía, Año 40 No. 120, pp. 207-237.

Lin, F. (2011). Is Earning management Opportunistic or Beneficial in Taiwan. International Journal of Economics and Finance, 3(1), 133-142. https://doi.org/10.5539/ijef.v3n1p133

Lisboa, I. (2016). Impact of financial crisis and family control on earning management of Portuguese listed firms. European Journal of Family Business, 6(2),118-131

Lombardi, R., Trequattrini, R, Cuozzo, B. y Cano-Rubio, M.(2019) Corporate Corruption Prevention, Sustainable Governance and Legislation: First Exploratory Evidence from the Italian Scenario, Journal of Cleaner Production, 217(20) https://doi.org/10.1016/j.jclepro.2019.01.214

López, P. (2006). Efectos Financieros del Gobierno Corporativo y ética en los negocios en México: El caso de Cemex y TV-Azteca. Revista Gerenciais, 5(1), 152-166.

Macías, A. y Román, F. (2014), Consecuencias económicas de la reforma de Gobierno Corporativo en un mercado de capitales emergente, El Trimestre económico, LXXXI (322), 357-412. https://doi.org/10.20430/ete.v81i322.117

Magrath L. y Weld, L.G. (2002). Abusive earnings management and early warning signs. The CPA Journal, 72, 50-53.

Mahmud, H. M. (2011). Sector-Wise Stock Return Analysis: An Evidence from Dhaka Stock Exchange in Bangladesh. International Journal of Business and Management 6(6): 276-285 https://doi.org/10.5539/ijbm.v6n6p276

McNichols, M. (2000). Research design issues in earning management studies. Journal of Accounting and Public Policy (19), 313-345. https://doi.org/10.1016/s0278-4254(00)00018-1

Melis A. (2004). Financial reporting, corporate communication and governance. Corporate Ownership and Control, 1(2), 31-37.

Menard, S. (2002). Quantitative Application in the Social Sciences, Thousand Oaks, London, New Delhi, Sage Publications Inc. https://doi.org/10.4135/9781412984867

Morck, R., Wolfenzon, D. and Yeung, B. (2005). Corporate governance, economic entrenchment, and growth. Journal of Economic Literature, 63, 655-720.

Mousa M. y Desoky, A. (2012). The association between internal governance mechanisms and corporate value: evidence from Bahrain. Asian Academy of management Journal of accounting and finance, (8), 67-91.

Nazir M.S. y Afza, T. (2018). Does managerial behavior of managing earnings mitigate the relationship between corporate governance and firm value? Evidence from an emerging market. Future Bunisess Journal, (4), 139-156.

Ning, Y. (2006). A different perspective of earnings management, Canadian Social Science, 2 (4), 53-59.

North, D. C. (1990). Institutions, Institutional Change and Economic Performance. New York: Norton.

OCDE (2004). White Paper sobre Gobierno Corporativo en América Latina. Centro para la cooperación con países no miembros de la OCDE.

OCDE (2015). G20/OECD Principles of Corporate Governance. Disponible en: https://mba.americaeconomia.com/sites/mba.americaeconomia.com/files/gobierno_corporativo.pdf

Peng, M. (2003). Institutional transitions and strategic choices. Academy of Management Review, 28(2), $275-296$. https://doi.org/10.2307/30040713

Poletti-Hughes, J. (2011). The value effect of corporate governance: evidence from Mexico. In: Global Conference on Business and Finance, Las Vegas. https://doi.org/10.2139/ssrn.1748624

Renders, A. y Gaeremynck, A. (2012). Corporate Governance, Principal-Principal Agency Conflicts, and firms Value in European Listed Companies. Corporate Governance: an International Review, 28 (2), 125-143. DOI: $10.1111 / \mathrm{j} .1467-8683.2011 .00900 . x$ 
Reyna, J.M., Durán, R. y Valdes, A.L. (2012). Corporate Governance, Ownership structure and performance in México. International Business Research, 5 (11).

Rodríguez, M. Cortez, K., Méndez, B. y Garza, H. (2015). Análisis de portafolio por sectores mediante el uso de algoritmos genéticos: caso aplicado a la Bolsa Mexicana de Valores. Contaduría y Administración, 60 (1), 87-112 https://doi.org/10.1016/s0186-1042(15)72148-0

Schipper, K. (1989). Commentary on earnings management. Accounting Horizons, 3(4), pp. 91-102.

Shleifer, A. and Vishny, R. (1997). A survey of corporate governance. Journal of Finance, 52, 737-83

Tangjitprom, N. (2013). Earning management and Corporate Governance in Thailand. National Institute of Development Administration \& Assumption University Bangkok, Thailand, 61(9), 41-45.

UK Corporate Governance Code (2017). The Financial Reporting Council Limited 2017, available at: www.frc.org.uk

Witt, M. A., \& Redding, G. (2013). Asian business systems: Institutional comparison, clusters and implications for varieties of capitalism and business systems theory. Socio-Economic Review, 11 (2), 265-300. http://dx.doi.org/10.2139/ssrn.2104088

Wright, M., Filatotchev, I., Hoskisson, R. and Peng, M. W. (2005). Strategy research in emerging economies: challenging the conventional wisdom. Journal of Management Studies, 42, 1-33. https://doi.org/10.1111/j.14676486.2005.00487.x

Young, M., Peng, M, Ahlstrom, D., Bruton, G., Jiang, Y. (2008). Corporate Governance in Emerging Economies: A Review of the Principal-Principal Perspective. Journal of management studies, 45(1), 196-220. https://doi.org/10.1111/j.1467-6486.2007.00752.x 